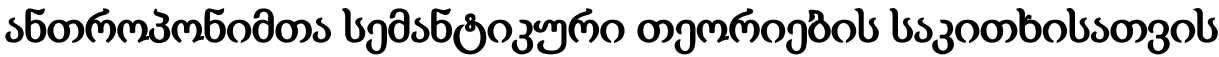

\section{бобм ззопозуды}

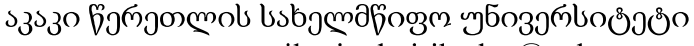
e-mail:ninokvirikadze@yahoo.com

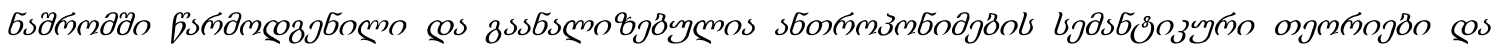

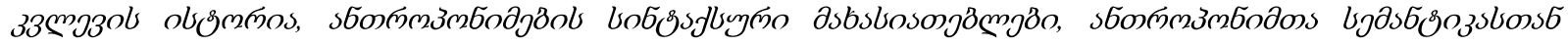

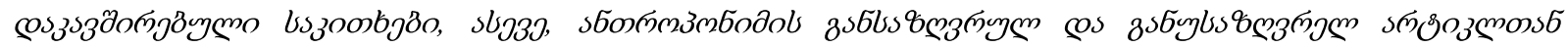

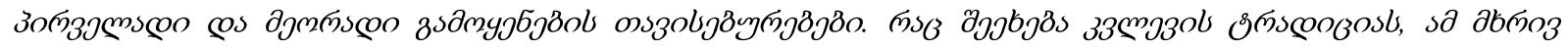

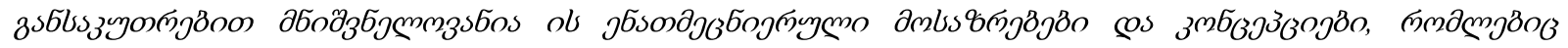

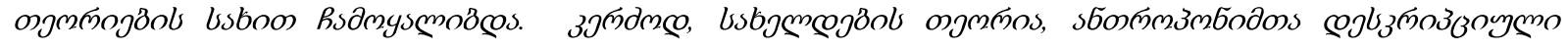

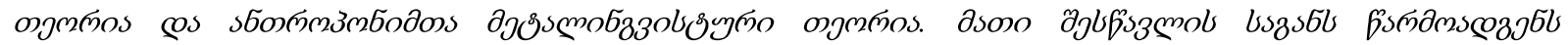

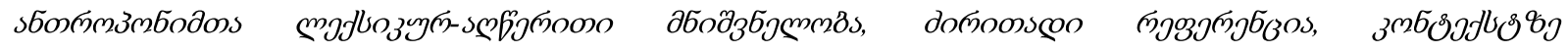

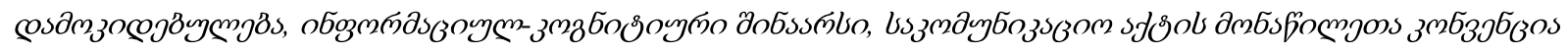
(упоолподวольбадљs).

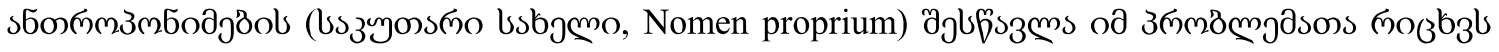

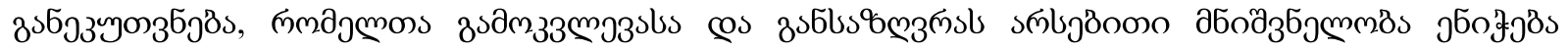

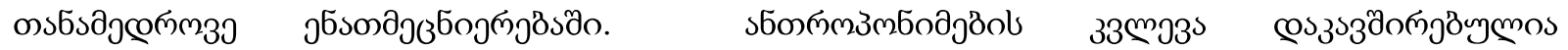

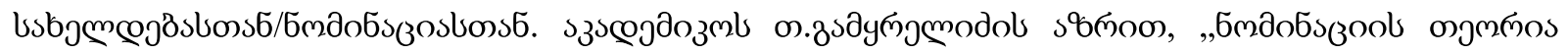

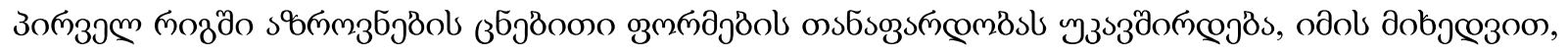

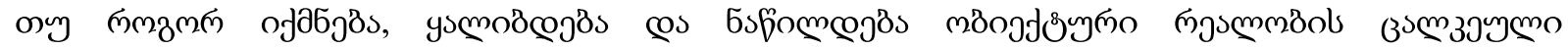

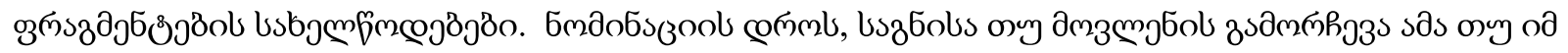

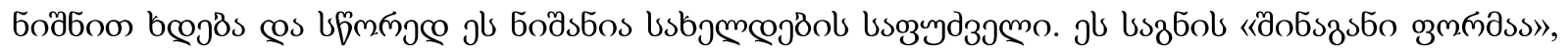

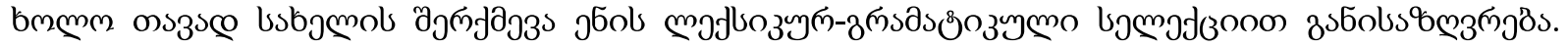

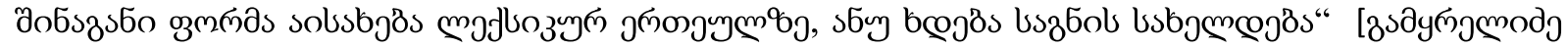
2008: 354].

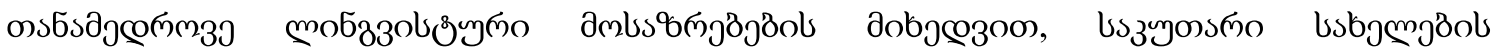

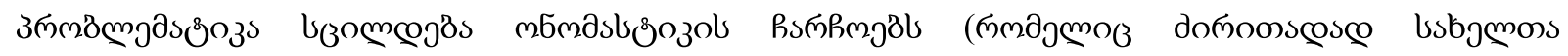




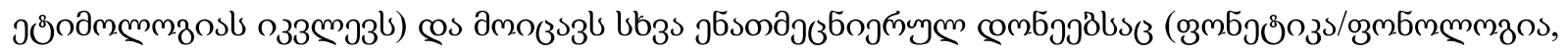

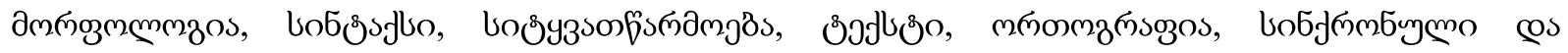

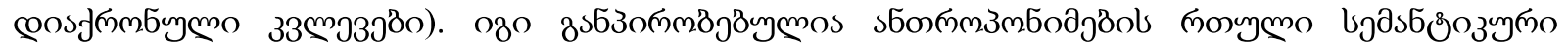

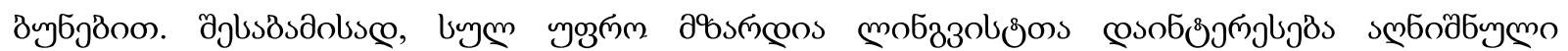

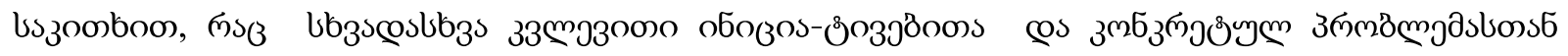

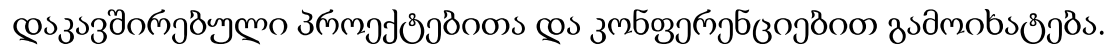

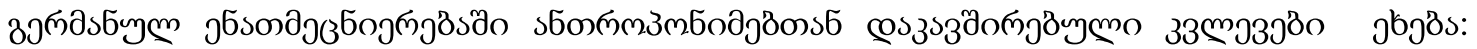

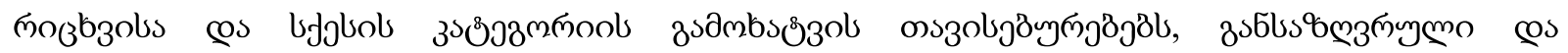

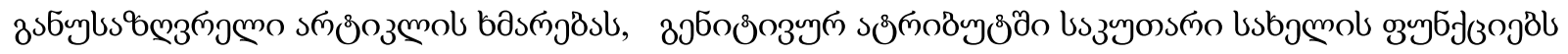

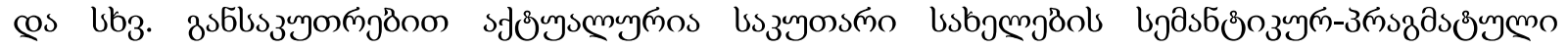

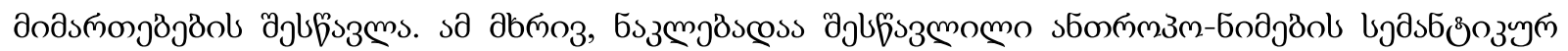

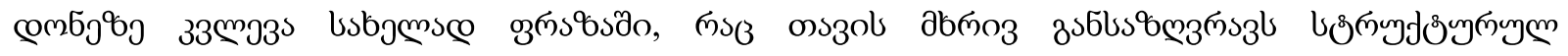

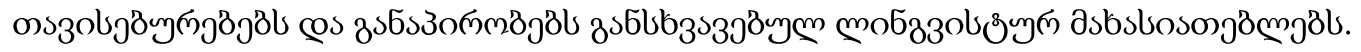

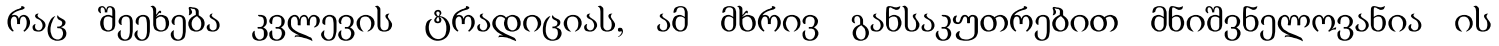

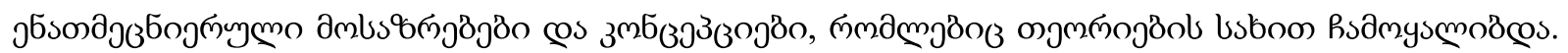

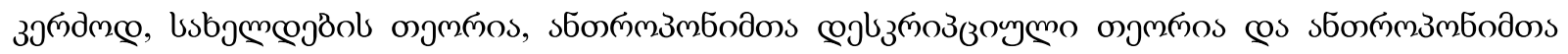

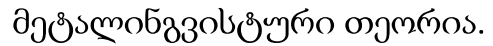

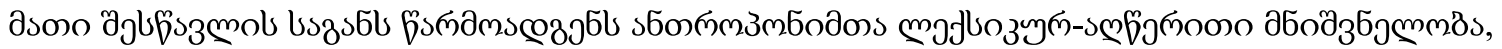

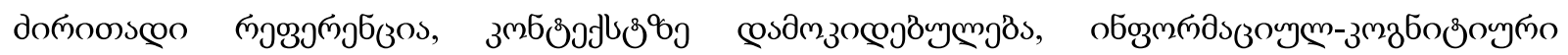

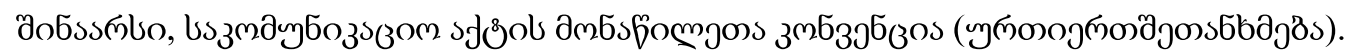

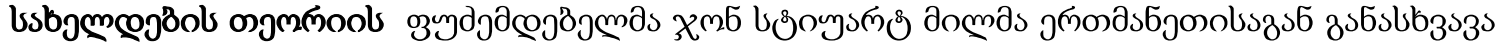

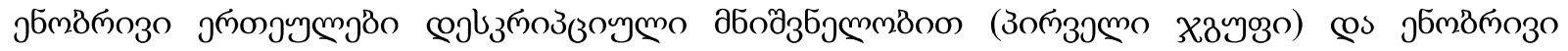

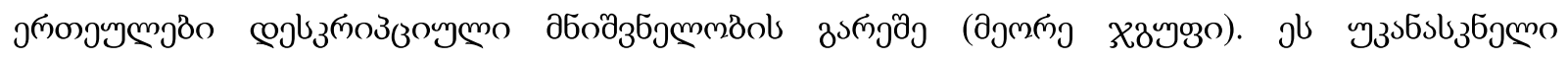

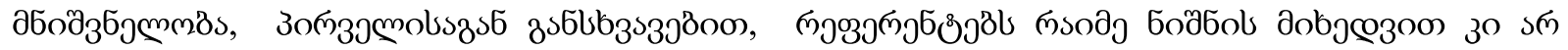

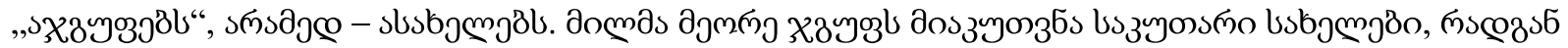

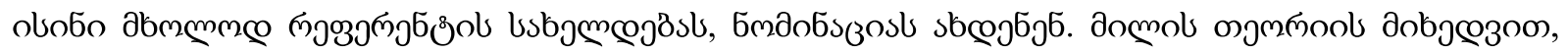

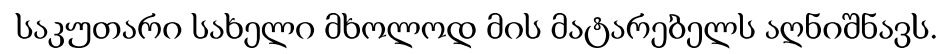

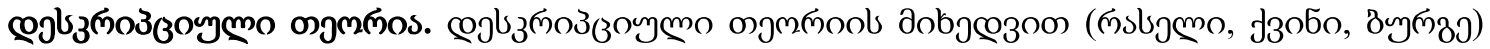

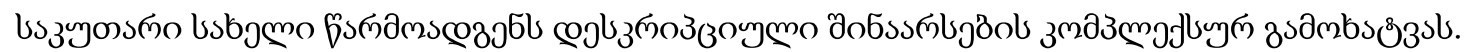

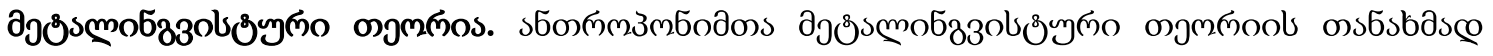

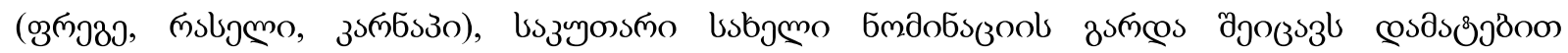

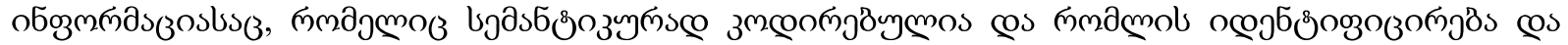




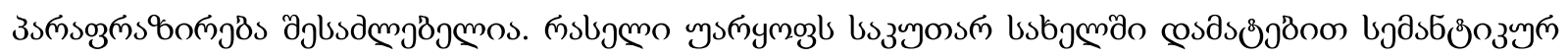

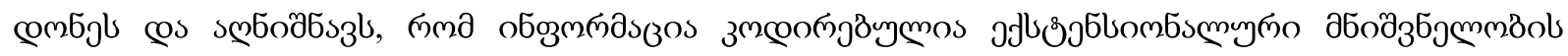

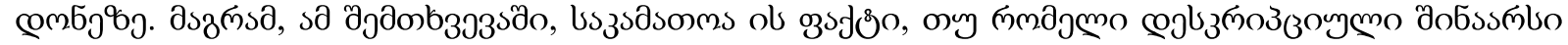

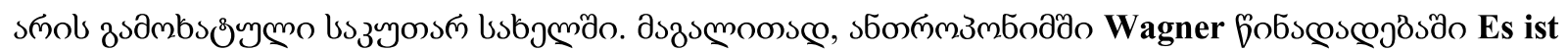

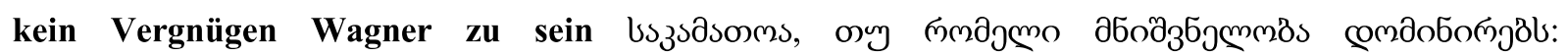

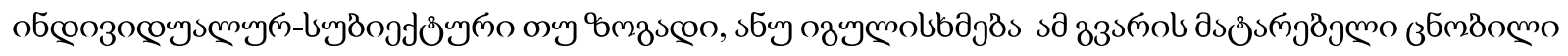

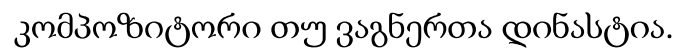

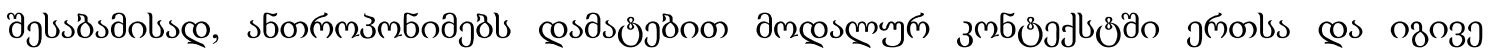

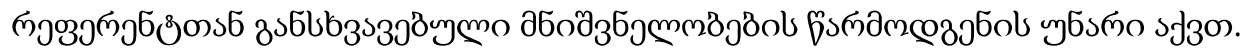

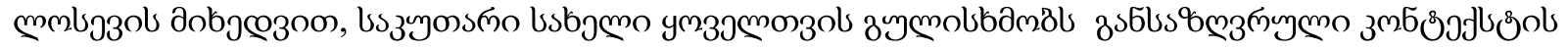

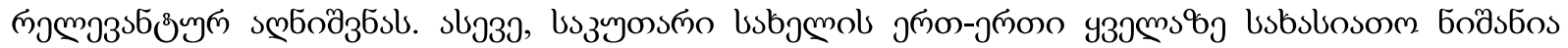

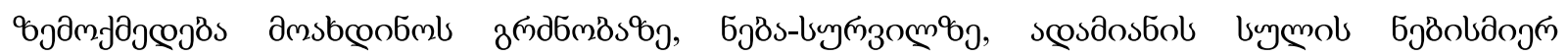

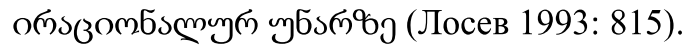

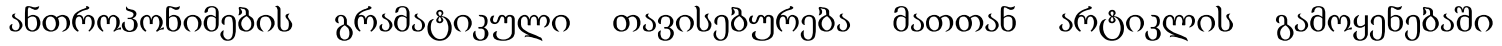

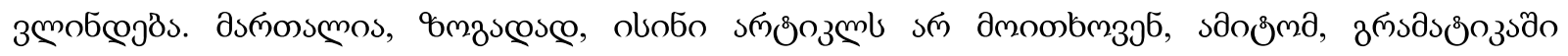

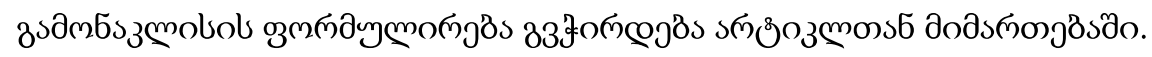

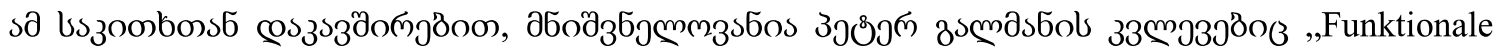

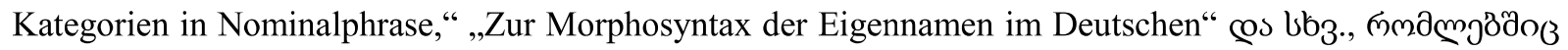

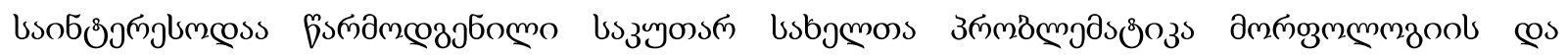

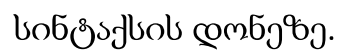

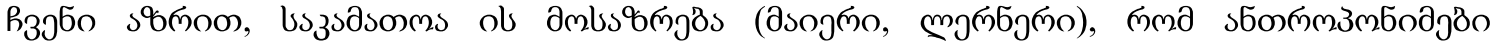

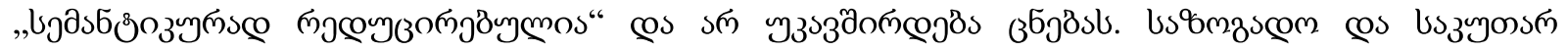

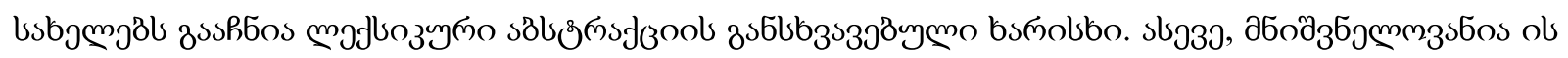

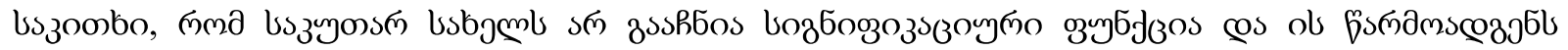

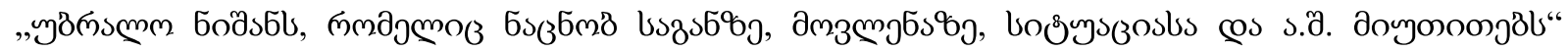
(Ахманова 1948: 8).

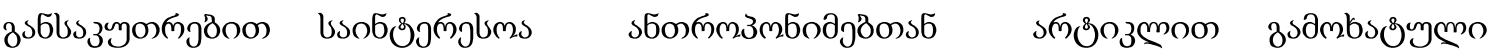

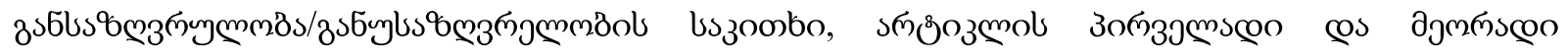

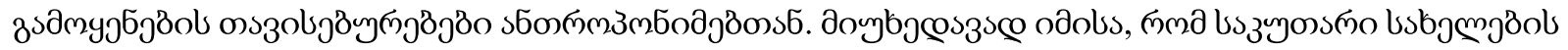

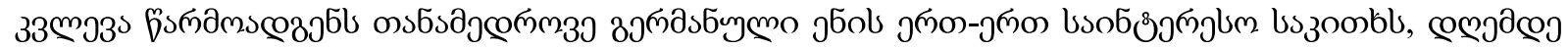

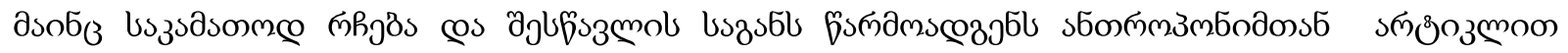

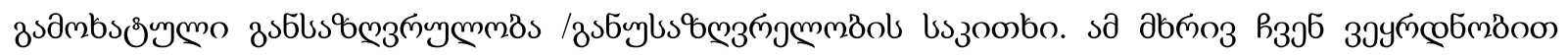




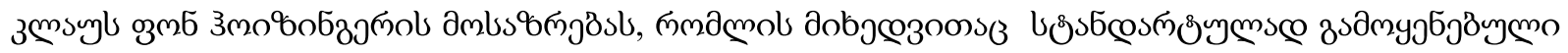

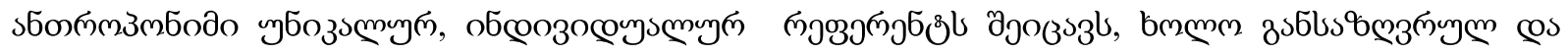
дыб

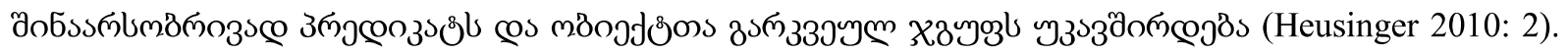

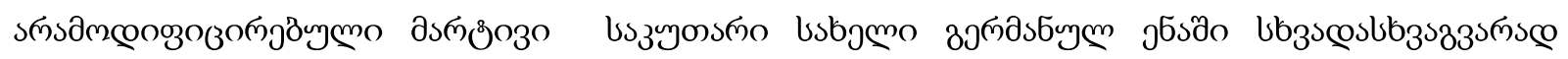

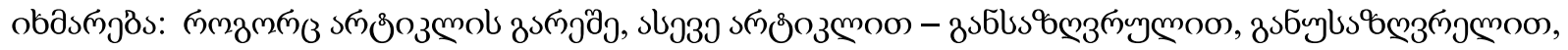

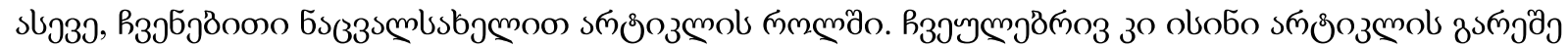

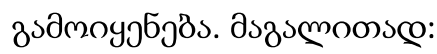

(1) a Selbst Mozart hätte das nicht viel besser machen können.

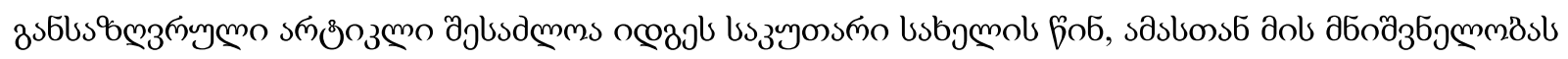

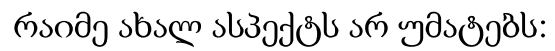

(1) b Selbst der Mozart hätte das nicht viel besser machen können.

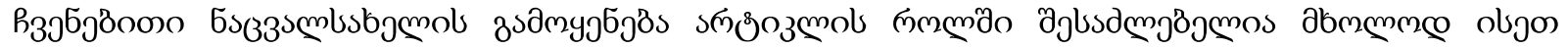

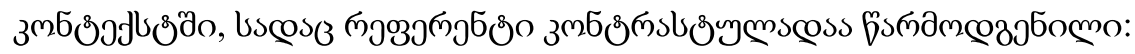

(1) c Selbst dieser Mozart hätte das nicht viel besser machen können.

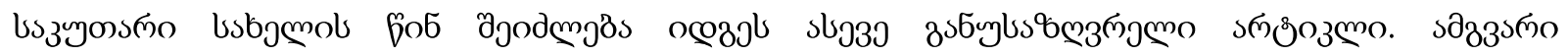

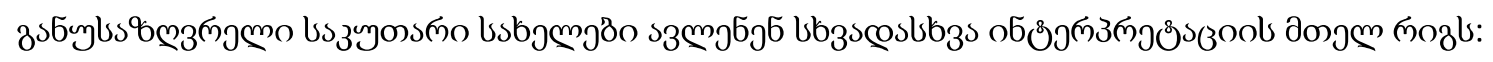

(1) d Selbst ein Mozart hätte das nicht viel besser machen können.

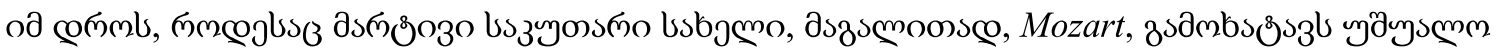

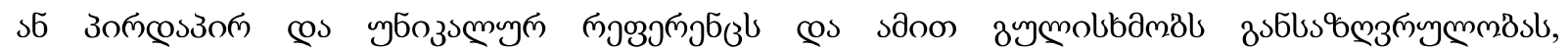

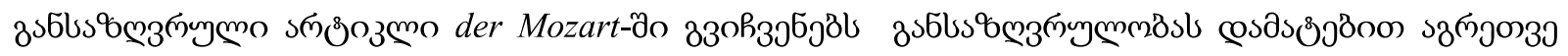

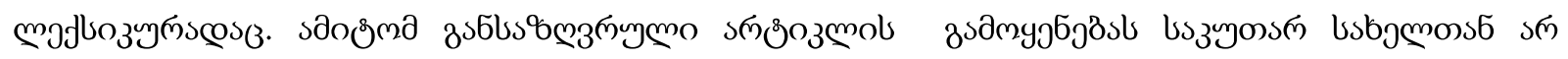

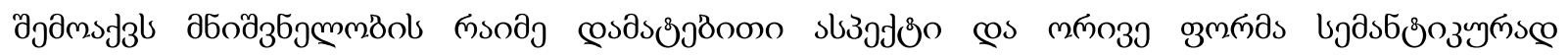

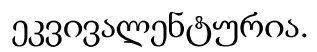

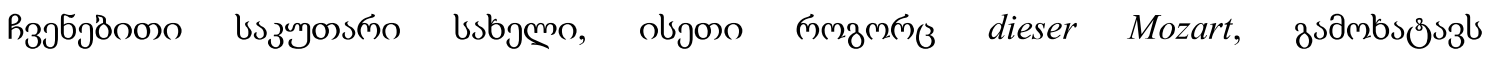

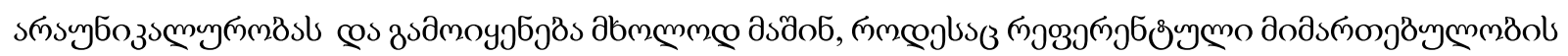

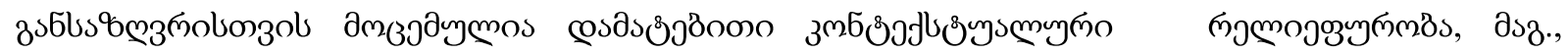

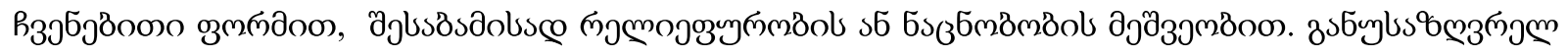

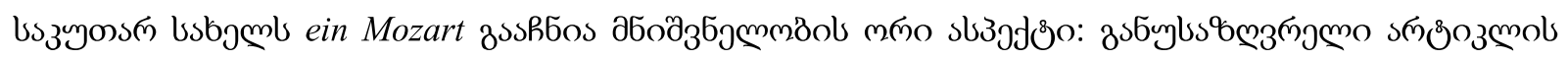

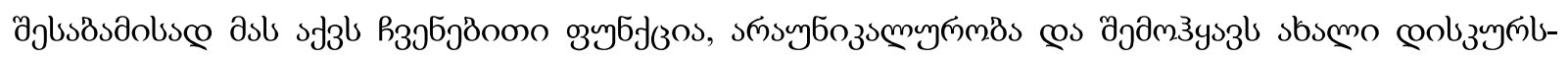

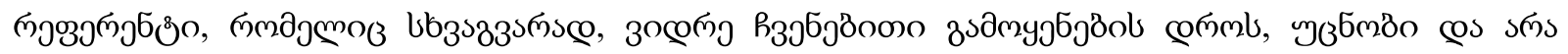

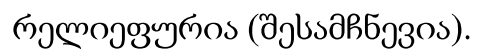




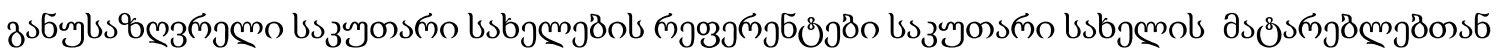

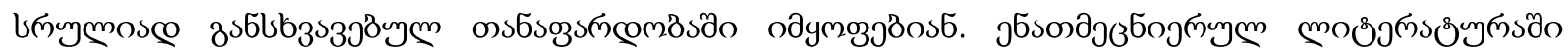

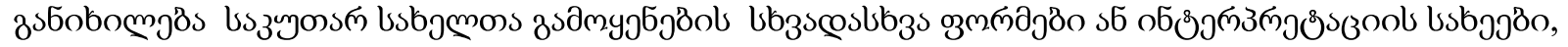

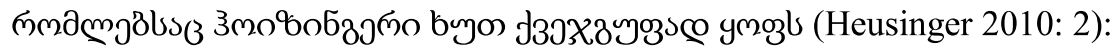

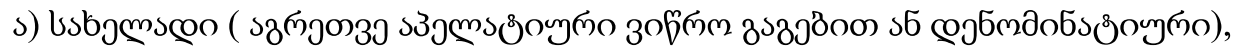

8) дэతిмбодоэ๓о,

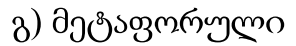

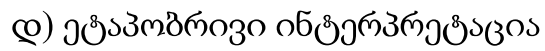

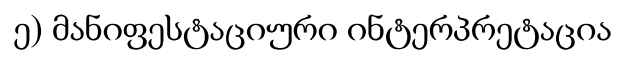

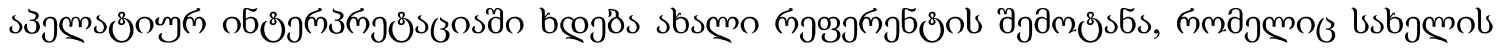

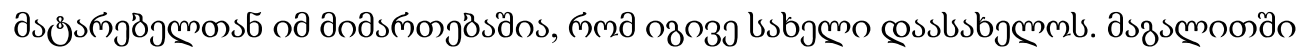

(2) Das Schicksal, ein Wagner zu sein (...) Er verharmlost nichts, aber er eifert auch nicht. Er erzählt von einer Familie, deren Mitglieder über mehr als 150 Jahre eine bestimmende Rolle im künstlerischen Leben Deutschlands gespielt haben, durch Abkunft privilegiert, aber mehr noch belastet. Sich davon durch Carr erzählen zu lassen ist eine Freude. Kein Vergnügen aber ist es offenbar, ein oder eine Wagner zu sein. ${ }^{1}$

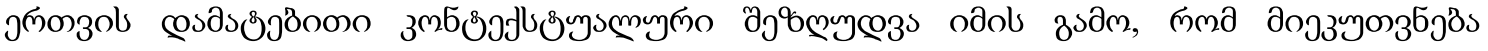

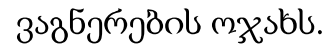

Weich und einlullend ist ihr Mozart, der behutsam zwischen Mezzoforte und Mezzopiano changiert. Ein Mozart, bei dem einem die Augen zufallen hätte es vorher keinen Kaffee gegeben. ${ }^{2}$

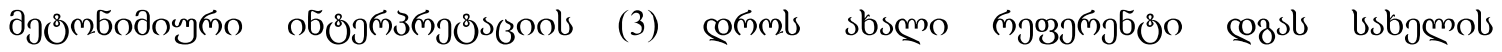

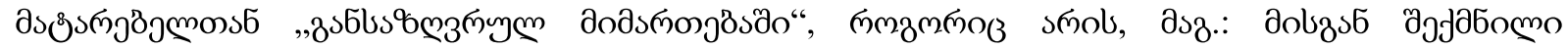

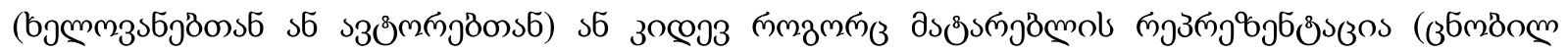

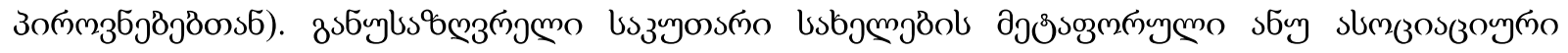

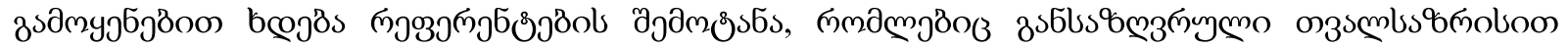

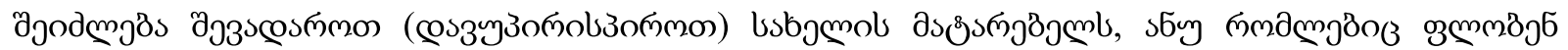

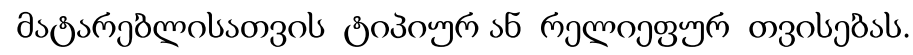

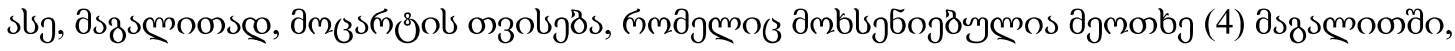

Der russische Pianist Jewgenij Kissin erobert das deutsche Publikum.

\footnotetext{
${ }^{1} \mathrm{http}: / /$ www.sueddeutsche.de/kultur/193/303188/text/4/ (25.1.2010).

${ }^{2} \mathrm{http}: / /$ magazin.klassik.com/konzerte/reviews.cfm?task=review\&PID=2011 (25.1.2010).
} 
Noch eine Oktoberrevolution: Am 10. Oktober 1971 kommt Jewgenij Kissin auf die Welt, ein Mozart aus Moskau. Innerhalb weniger Jahre katapultierte sich dieser Pianist an die Spitze der Weltklangliste. ${ }^{1}$

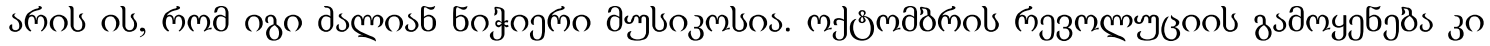

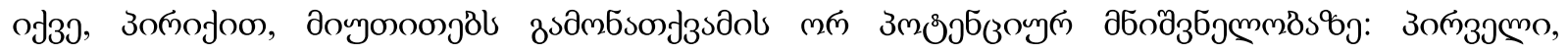

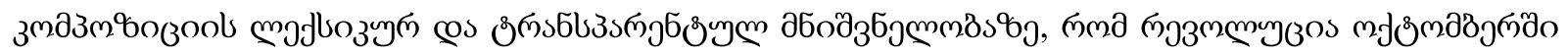

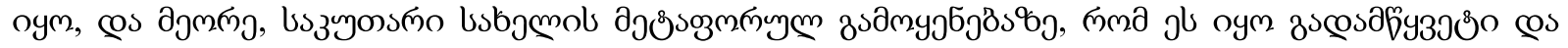

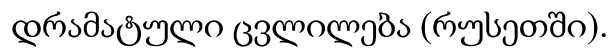

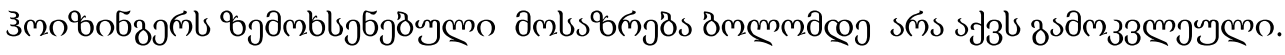

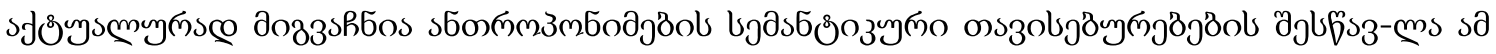

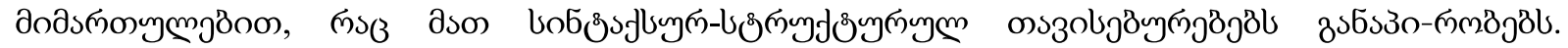

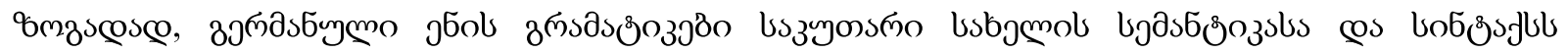

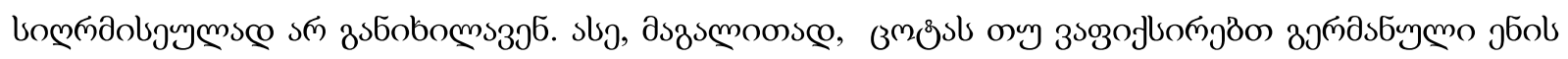

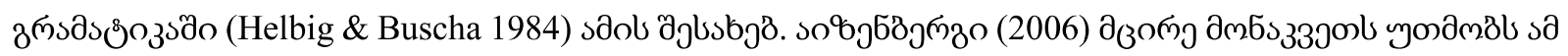

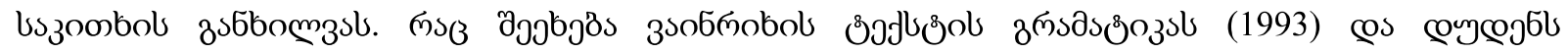

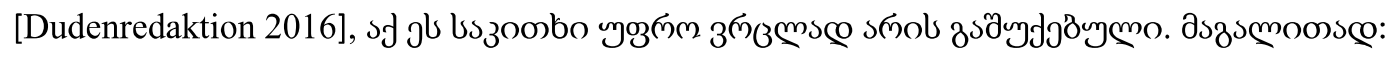

Eigennamen sind „,aus sich heraus“ definit: Sie stehen für eine besondere Person oder Sache und sind allgemein oder zumindest einem bestimmten Personenkreis bekannt. Der Artikel ist daher bei Eigennamen nicht wirklich wählbar - was nicht heißt, dass er immer vorhanden ist. Im Einzelnen kann man bei den Eigennamen vielmehr folgende Gebrauchsweisen des definiten Artikels unterscheiden: (i) primäre Artikellosigkeit, (ii) primärer Artikelgebrauch, (iii) sekundärer Artikelgebrauch, (iv) sekundäre Artikellosigkeit (Duden 2016: 307, § 396 ff.).

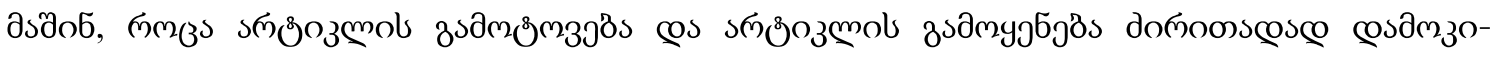

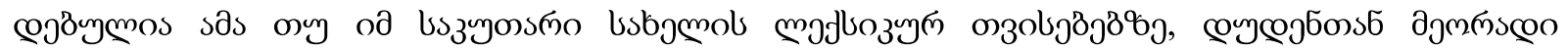

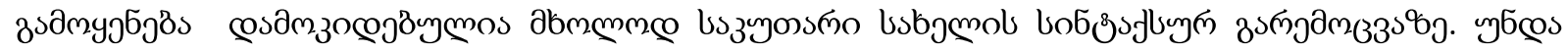

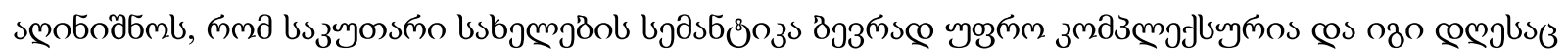

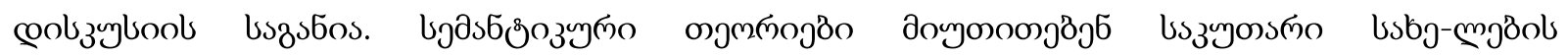

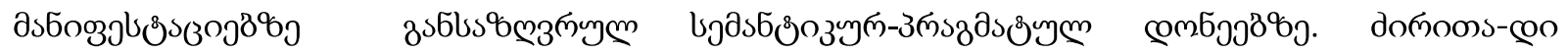

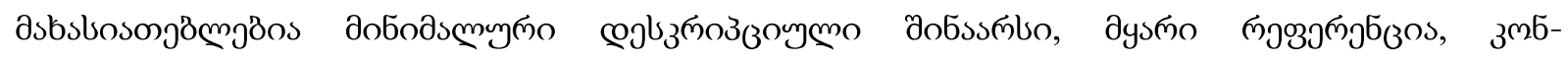

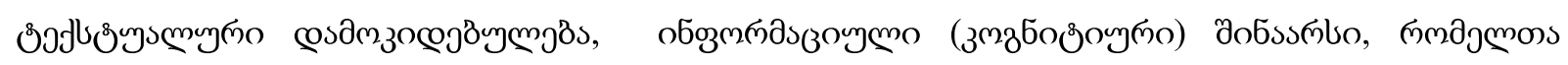

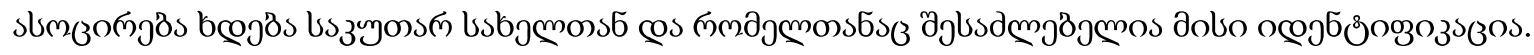

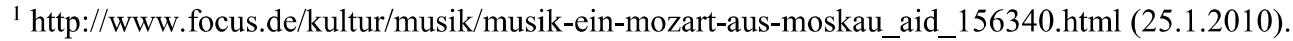




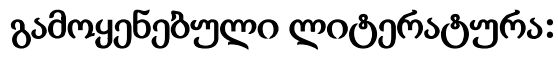

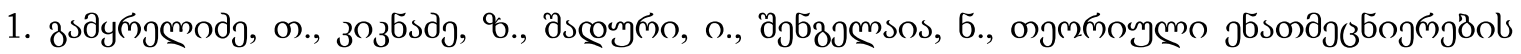

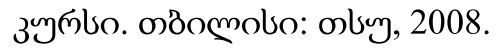

2. Duden. Hg. von der Dudenredaktion. Der Große Duden. Band 4. Die Grammatik. 9. völlig neu erarbeitete und erw. Auflage. Mannheim,Wien, Zürich: Dudenverlag 2005. § 396 ff. 307. 2016.

3. Glück, Helmut und Rödel, Michael (Hg.) Metzler Lexikon Sprache. 5., aktualisierte und überarbeitete Auflage. Stuttgart: J. B. Metzler Verlag GmbH 2016.

4. Heusinger, Klaus von, Zur Grammatik indefiniter Eigennamen. Zeitschrift für Germanistische Linguistik, Stuttgart, Januar 2010.

5. Heusinger, Klaus von, Salienz und Referenz. Der Epsilonoperator in der Semantik der ${ }^{\text {Nominalphrase }}$ und anaphorischer Pronomen. Studia grammatica 43. Berlin: Akademie Verlag 1997.

6. Heusinger, Klaus von \& Wespel, Johannes, Indefinite Proper Names and Quantification over Manifestations. In: E. Puig-Waldmüller (ed.). Proceedings of Sinn und Bedeutung 11. Barcelona: Universitat Pompeu Fabra 2007. 332-345.

7. Heusinger, Klaus von \& Wespel, Johannes, Indefinite Eigennamen in generischen Sätzen. Quantifikation über Manifestationen von Individuen. Linguistische Berichte 217. 2009. 5-38.

8. Ronneberger-Sibold, Elke, Warennamen. In: A. Brendler \& S. Brendler (eds.). Namenarten und ihre Erforschung. Ein Lehrbuch für das Studium der Onomastik. Hamburg: Baar 2004, 557-603.

9. Weinrich, Harald, Textgrammatik der deutschen Sprache. Mannheim: Dudenverlag 1993.

10. Ахманова, О.С., К вопросу о слове в языке и речи // Доклады и сообщения филологического факультета МГУ. Москва: МГУ, 1948.

11. Лосев, А.Ф., Бытие. Имя. Космос. Москва: Мысль, 1993. 
Nino Kvirikadze

Akaki Tsereteli State University

\title{
For the Question of the Semantic Theories of Antroponyms
}

\begin{abstract}
The article presents and analyzes the semantic theories and the history of the study of anthroponyms, the syntactic characteristics of anthroponyms, issues related to the semantics of anthroponyms, as well as the features of the primary and secondary use of anthroponyms with a definite and indefinite article. As far as the tradition of research is concerned, linguistic considerations and concepts developed in the form of theories are especially important in this regard. In particular, the theory of names, the descriptive theory of anthroponyms, and the metallinguistic theory of anthroponyms. The subject of their research is the lexical and descriptive meaning of anthroponyms, the main reference, dependence on the context, information and cognitive content, convention (mutual agreement) of the participants in the communicative act.
\end{abstract}

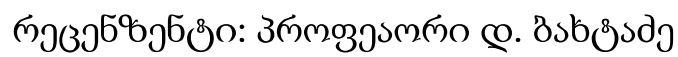

